\title{
Understanding cancer lineage plasticity: reversing therapeutic resistance in metastatic prostate cancer
}

\author{
“...an increasingly accepted mechanism of resistance in approximately a \\ quarter of tumors involves lineage plasticity.”
}

First draft submitted: 2 March 2017; Accepted for publication: 6 March 2017; Published online: 4 May 2017

Keywords: androgen receptor • castrate-resistant prostate cancer • epigenetic therapy • EZH2 - lethal prostate cancer $\bullet$ lineage plasticity $\bullet$ neuroendocrine $\bullet$ prostate cancer metastasis

Prostate cancer $(\mathrm{PCa})$ is among the most commonly diagnosed cancers in men throughout westernized countries and is a leading cause of cancer-related mortality [1]. Androgen-deprivation therapy (ADT) has been the backbone for $\mathrm{PCa}$ treatment by inhibiting androgen receptor (AR) signaling. However, resistance to this therapy is inevitable and men will progress to castrateresistant PCa (CRPC). Although most patients will undergo resistant mechanisms involving the restoration of AR signaling and maintenance of an adenocarcinoma phenotype (CRPC-Ad), an increasingly accepted mechanism of resistance in approximately a quarter of tumors involves lineage plasticity. These tumors express from low to absent AR levels, often demonstrating neuroendocrine features (CRPC-NE) and are indifferent to AR signaling. Further, genomic analysis reveals that CRPC-NE evolves from CRPC-Ad [2], but currently the molecular mechanisms underlying CRPC-NE evolution are not completely understood. Both clinical and preclinical data are emerging to where it is now possible to begin understanding molecular mechanisms driving resistance to antiandrogen therapy via lineage plasticity.
Data from independent investigations have started to shed light on driver genetic events governing the progression of CRPC-Ad to CRPC-NE, and how molecular alterations contribute to therapy resistance via tumor evolution. An initial study investigating the status of RB1, TP53 and $P T E N$ tumor suppressor genes in prostate adenocarcinomas and small-cell PCa (SCPC) subtypes revealed that combined alterations in these three tumor suppressor genes was a common occurrence. A major observation by Tan et al., was that deletion of $R B 1$ was a common event in AR negative $S C P C$, but not in high-grade AR positive adenocarcinomas, suggesting RB1 deletion is critical for progression of SCPC [3]. Aparicio et al. also reported in independent studies, that combined alterations in RB1, TP53 and/or PTEN genes were more frequent in CRPC-NE tumors when compared to CRPC-Ad tumors, demonstrating that indeed CRPC-NE tumors share molecular features with SCPC [4]. This was further validated by more recent studies in human samples that showed progression of CRPC-Ad to a CRPC-NE phenotype involved the acquisition of alterations in

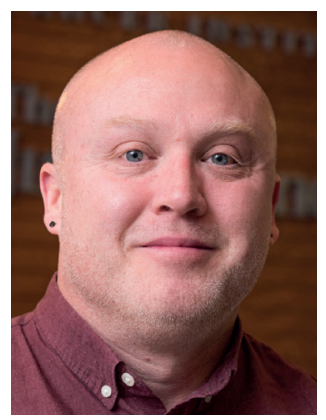

Leigh Ellis

Department of Oncologic Pathology, Dana-Farber Cancer Institute, Brigham \& Women's Hospital, Harvard Medical School, 450 Brookline Avenue, Boston, MA 02215, USA

and

Department of Pathology, Brigham \& Woman's Hospital, Harvard Medical School, Boston, MA 02115, USA leigh_ellis@dfci.harvard.edu 
RB1 and TP53 [5,6]. The concept that partnering mutations are required for development of SCPC or CRPC-NE has been significantly supported by genetically engineered mouse models. This was initially demonstrated by the generation of the transgenic adenocarcinoma mouse prostate murine model which overexpresses an SV40 transgene specifically

\section{"...further evaluation of alisertib in patients with CRPC-NE as single-agent and combination approaches is waranted.}

in the prostatic tissue of animals [7]. The resulting tumors are largely due to transgene-mediated attenuation of $R b 1$ and $T p 53$ expression/function. Later, a more direct mouse model was generated involving specific codeletion of $R b 1$ and Tp53 [8]. Both models result in progression to metastatic disease and resistance to castration via progression to a prostate neuroendocrine phenotype.

Apart from mutations occurring in key tumor suppressor genes, additional genes have been identified as potential key regulators of CRPC-NE and may offer novel molecular targets for therapeutic interventions. Overexpression and gene amplification of MYCN (N-MYC) and AURKA (Aurora-A) is a significant occurrence in human CRPC-NE [9,10]. In preclinical human cell lines and mouse models, it has now been well demonstrated that N-MYC is associated in the progression to CRPC-NE [9,11]. Interestingly, stabilization of N-MYC can be achieved via dimerization with Aurora-A, which can be targeted using allosteric Aurora-A inhibitors [12,13]. Targeting of Aurora-A with an allosteric inhibitor (alisertib) in preclinical neuroendocrine prostate cancer (NPEC) models demonstrated significant anti-tumor activity associated with degradation of N-MYC [11]. More recently, Phase II clinical data reported substantial single-agent activity with alisertib treatment of patients with CRPC-NE (Beltran, NCT01799278), though a Phase I/II trial of alisertib in combination with abiraterone and prednisone yielded low activity in patients with metastatic castrate-resistant prostate cancer (mCRPC) [14]. Selection of patients with CRPC-NE was performed by Beltran et al., which may explain the reported differences in patient responses. Nevertheless, further evaluation of alisertib in patients with CRPC-NE as single-agent and combination approaches is warranted.

Recently, the role of $R B 1$ toward cellular reprogramming and governing of pluripotency networks was reported [15]. This investigation utilized induced pluripotent stem-cell (iPSC) reprogramming as a cellular model to demonstrate that $R B 1$ regulates reprogramming of fibroblasts to iPSCs. It was found that $R B 1$ globally represses pluripotency networks, which renders cells to be more amendable to reprogramming via deregulation of factors like $S O X 2$. This intriguing finding is consistent to two more recent publications. $\mathrm{Mu}$ et al., by engineering knockdown of $R B 1$ and TP53 in a human PCa cell line that overexpresses AR (LNCaP-AR), a model of CRPC-Ad, demonstrated that only dual knockdown of both genes caused marked resistance to antiandrogen treatment. Also, cells lacking RB1 and TP53 showed lineage plasticity, with dramatic loss in expression of luminal epithelial cell markers and increased expression of basal epithelial and neuroendocrine cell markers [16]. In correlation to the study by Kareta et al., SOX2 expression was induced by silencing of RB1 and TP53 in LNCaP-AR cells, and interestingly, expression of basal and neuroendocrine markers was dependent on SOX2 expression. In addition, using a mouse model of prostate adenocarcinoma (AR expressing) driven by the deletion of Pten, co-deletion of Rb1 resulted in aggressive metastatic disease with heterogeneous histology expressing both markers of adenocarcinoma and neuroendocrine disease, where variable AR expression was observed [17]. Animals displayed transient response to castration, with all progressing to castrate-resistant disease. Few animals contained Tp53 mutations, suggesting an important role for loss of $T p 53$ toward castration resistance. Gene expression analysis revealed that loss of $R b 1$ shifted tumors to a phenotype with significant overlap of genes observed in human neuroendocrine PCa. Most relevant where notable changes in neuroendocrine lineage genes, and genes related to stemness and epigenetic reprogramming, including Sox2 and Ezh2. Excitingly, Ku et al. demonstrated that Ezh2 inhibition increased levels of $\mathrm{AR}$ and restored sensitivity to antiandrogen therapy. Further, the importance of Ezh2 as a key mediator of PCa lineage switching was recently shown in an independent study. Using the same prostate adenocarcinoma mouse model driven by loss of Pten, the concurrent overexpression of $M y c N$ initiated progression to neuroendocrine $\mathrm{PCa}$ with reduced overall AR expression [11]. Like the study presented by $\mathrm{Ku}$ et al., overexpression of $\mathrm{MycN}$ resulted in increased expression of Ezh2 and chromatin redistribution. Further, $N M y c$ expression sensitizes cells to Ezh2 inhibitors. These independent studies suggest mechanistic importance of Ezh2 mediating progression to CRPC-NE and use of Ezh2 inhibitors in combination with other therapies, including antiandrogen therapy to treat patients who have progressed to CRPC-NE.

Clinical data comparing human CRPC-Ad with CRPC-NE tumors also highlight significant deregulation of stemness/epigenetic regulators in 
CRPC-NE [9,18]. Specifically, data from Clermont et al. demonstrated significant increased expression of numerous chromatin modifiers in NPEC samples including DNMT3A, DMNT3B and DMNT1 involved in DNA methylation, and histone modifying enzymes including EZH2, CBX8 and DOT1L [18]. By overlapping gene transcripts across multiple independent preclinical and clinical NPEC samples, the development of a 185-gene list referred to as 'neuroendocrine-associated repression signature' (NEARS) demonstrated significant enrichment of polycomb (involving EZH2) targeted genes [18]. NEARS selected patients with rapid development of metastatic disease, further validating polycomb action in development of lethal PCa. The development of gene signatures, like NEARS, from noninvasive sources including cell-free DNA/RNA or circulating tumor cells (CTCs) is of high importance for clinical use. These signatures may enable earlier detection of CRPC-Ad switching to CRPC-NE. Recent evaluation of CTCs from CRPC-NE patients compared with patients with CRPC-Ad revealed that CTCs from CRPC-NE patients carry unique characteristics [19]. More recently, exome sequencing comparing a patients metastatic tumor sample and CTCs was reported. Excitingly, this longitudinal analysis demonstrated the development of resistance to ADT via tumor evolution involving acquisition of neuroendocrine features involving a nonsense mutation of the RB1 gene [20]. Such analysis increases confidence in the use of CTCs to track tumor evolution and selection of treatment options for patients progressing with ADT.

Moving forward, significant advances have been made in PCa modeling including genetically modified mouse models, patient-derived xenografts, mouse and human 3D organoid models and availability of patient samples. These combined with genomic and proteomic technology will allow for a more rapid gain of knowledge into understanding the genetic and epigenetic interplay which drive lineage plasticity and therapy resistance. Overall, CRPC-NE tumors are significantly more epigenetically deregulated compared with CRPC-Ad tumors. To date, a large focus has been directed towards Ezh2 action, and clinically relevant inhibitors of Ezh2 have proven effective in reversing ADT resistance in pre-clinical studies [17]. Our data aligned with independent studies, position Ezh2 as a primary epigenetic target to initialize clinical trials in CRPC-NE patients. In addition, alternate epigenetic/chromatin modifiers including the Switch/Sucrose Non-Fermentable (SWI/SNF) [21,22] complex and Bromo- and Extra-Terminal domains [Ellis Lab, Unpublished Data] need to be further tested in models of CRPC-NE. This continued growth in knowledge will ultimately allow for the implementation of targeted/epigenetic therapies to inhibit or reverse lineage plasticity and generate sustainable clinical responses in patients with CRPC-NE.

\section{Financial \& competing interests disclosure}

The authors have no relevant affiliations or financial involvement with any organization or entity with a financial interest in or financial conflict with the subject matter or materials discussed in the manuscript. This includes employment, consultancies, honoraria, stock ownership or options, expert testimony, grants or patents received or pending, or royalties.

No writing assistance was utilized in the production of this manuscript.

\section{References}

1 Siegel RL, Miller KD, Jemal A. Cancer statistics, 2015. CA Cancer J. Clin. 65(1), 5-29 (2015).

2 Wang HT, Yao YH, Li BG, Tang Y, Chang JW, Zhang J. Neuroendocrine Prostate Cancer (NEPC) progressing from conventional prostatic adenocarcinoma: factors associated with time to development of NEPC and survival from NEPC diagnosis-a systematic review and pooled analysis. J. Clin. Oncol. 32(30), 3383-3390 (2014).

3 Tan HL, Sood A, Rahimi HA et al. Rb loss is characteristic of prostatic small cell neuroendocrine carcinoma. Clin. Cancer Res. 20 (4), 890-903 (2014).

4 Aparicio AM, Shen L, Tapia EL et al. Combined tumor suppressor defects characterize clinically defined aggressive variant prostate cancers. Clin. Cancer Res. 22(6), 1520-1530 (2016).

5 Kumar A, Coleman I, Morrissey C et al. Substantial interindividual and limited intraindividual genomic

diversity among tumors from men with metastatic prostate cancer. Nat. Med. 22(4), 369-378 (2016).

6 Beltran H, Prandi D, Mosquera JM et al. Divergent clonal evolution of castration-resistant neuroendocrine prostate cancer. Nat. Med. 22(3), 298-305 (2016).

7 Gingrich JR, Barrios RJ, Morton RA et al. Metastatic prostate cancer in a transgenic mouse. Cancer Res. 56(18), 4096-4102 (1996).

8 Zhou Z, Flesken-Nikitin A, Corney DC et al. Synergy of p53 and Rb deficiency in a conditional mouse model for metastatic prostate cancer. Cancer Res. 66(16), 7889-7898 (2006).

9 Beltran H, Rickman DS, Park K et al. Molecular characterization of neuroendocrine prostate cancer and identification of new drug targets. Cancer Discov. 1(6), 487-495 (2011).

10 Mosquera JM, Beltran H, Park K et al. Concurrent $A U R K A$ and $M Y C N$ gene amplifications are harbingers of 
lethal treatment-related neuroendocrine prostate cancer. Neoplasia 15(1), 1-10 (2013).

11 Dardenne E, Beltran H, Benelli $\mathrm{M}$ et al. N-Myc induces an $E Z H 2$-mediated transcriptional program driving neuroendocrine prostate cancer. Cancer Cell 30 (4), 563-577 (2016).

12 Brockmann M, Poon E, Berry T et al. Small molecule inhibitors of aurora-a induce proteasomal degradation of $\mathrm{N}$-myc in childhood neuroblastoma. Cancer Cell 24(1), 75-89 (2013).

13 Gustafson WC, Meyerowitz JG, Nekritz EA et al. Drugging $M Y C N$ through an allosteric transition in Aurora kinase A. Cancer Cell 26(3), 414-427 (2014).

14 Lin J, Patel SA, Sama AR et al. A Phase I/II study of the investigational drug alisertib in combination with abiraterone and prednisone for patients with metastatic castration-resistant prostate cancer progressing on abiraterone. Oncologist 21(11), 1296e-1297e (2016).

15 Kareta MS, Gorges LL, Hafeez $S$ et al. Inhibition of pluripotency networks by the $\mathrm{Rb}$ tumor suppressor restricts reprogramming and tumorigenesis. Cell Stem Cell 16(1), 39-50 (2015).
16 Mu P, Zhang Z, Benelli M et al. SOX2 promotes lineage plasticity and antiandrogen resistance in TP53- and RB1deficient prostate cancer. Science 355(6320), 84-88 (2017).

$17 \mathrm{Ku}$ SY, Rosario S, Wang Y et al. Rb1 and $\operatorname{Trp} 53$ cooperate to suppress prostate cancer lineage plasticity, metastasis, and antiandrogen resistance. Science 355(6320), 78-83 (2017).

18 Clermont PL, Lin D, Crea F et al. Polycomb-mediated silencing in neuroendocrine prostate cancer. Clin. Epigenetics 7(1), 40 (2015).

19 Beltran H, Jendrisak A, Landers $M$ et al. The initial detection and partial characterization of circulating tumor cells in neuroendocrine prostate cancer. Clin. Cancer Res. 22(6), 1510-1519 (2016).

20 Lack J, Gillard M, Cam M, Paner GP, Vanderweele DJ. Circulating tumor cells capture disease evolution in advanced prostate cancer. J. Transl. Med. 15(1), 44 (2017).

21 Lee RS, Roberts CW. Linking the SWI/SNF complex to prostate cancer. Nat. Genet. 45(11), 1268-1269 (2013).

22 Prensner JR, Iyer MK, Sahu A et al. The long noncoding RNA SChLAP1 promotes aggressive prostate cancer and antagonizes the SWI/SNF complex. Nat. Genet. 45(11), 1392-1398 (2013). 